\title{
Sex Chromatin in Peripheral Blood Neutrophils and Sex Determination
}

\author{
Zoja Miknienè, Rita Ivanauskaitė \\ Lithuanian University of Health Sciences, Kaunas, Lithuania \\ Email: zoja.mikniene@lsmuni.lt, * rita.ivanauskaite@yahoo.com
}

How to cite this paper: Mikniene, Z. and Ivanauskaite, R. (2018) Sex Chromatin in Peripheral Blood Neutrophils and Sex Determination. Case Reports in Clinical Medicine, 7, 55-62.

https://doi.org/10.4236/crcm.2018.71005

Received: November 24, 2017

Accepted: January 15, 2018

Published: January 18, 2018

Copyright $\odot 2018$ by authors and Scientific Research Publishing Inc. This work is licensed under the Creative Commons Attribution International License (CC BY 4.0).

http://creativecommons.org/licenses/by/4.0/

(c) (i) Open Access

\begin{abstract}
The objective of this paper is to review in what forms sex chromatin can appear in peripheral blood neutrophils and how sex determination can be done using sex chromatin appendages. Sex chromatin is an approximately 1 micron clump of chromatin seen usually at the periphery of female nuclei in certain tissues and called "Barr body" and as a drumstick in polymor phonuclear neutrophils nuclei in the blood smears. Sex chromatin is derived from one of the two $\mathrm{X}$ chromosomes in the female which replicates its deoxyribonucleic acid much later than the other and is thus positively heteropyknotic. In 1954, Davidson and Smith were the first to identify and report the presence of neutrophil drumsticks and nonspecific appendages and their differences in sexes. The inactive $\mathrm{X}$ chromosome in neutrophils appears in one of the five forms: drumsticks, racquet forms, sessile nodules, small clubs and minor lobes. Only drumstick appendage is sex-specific and considered for sex diagnosis. For sex determination, drumsticks are significantly higher in females than in males $(\mathrm{p}<0.001)$.
\end{abstract}

\section{Keywords}

Sex Chromatin, Drumstick, Neutrophil, Blood, Sex Determination

\section{Introduction}

Sex chromatin is an approximately 1 micron clump of chromatin seen usually at the periphery of female nuclei in certain tissues like corneal epithelium, buccal mucosa, oral and vaginal mucosa, fibroblasts etc. and called "Barr body" and as a drumstick in polymorphonuclear neutrophils nuclei in the blood smears [1] [2]. Sex chromatin was found in all tissues except in liver and pancreatic acinar cells [3].

In 1954, Davidson and Smith were the first to identify and report the presence 
of neutrophil drumsticks and nonspecific appendages and their differences in sexes and sometimes drumstick appendages are called "Davidson's bodies" [1] [3]-[8]. The frequencies and the distribution of these appendages were not influenced by sex only, but also by many other factors such as hormones, granulocytes metabolism, cell proliferation, and age [4] [8].

\section{Origination}

Sex chromatin is derived from one of the two $\mathrm{X}$ chromosomes in the female which replicates its deoxyribonucleic acid much later than the other and is thus positively heteropyknotic [1] [2] [3]. It leads to condensation of the inactive $\mathrm{X}$ chromosome into the heterochromatic sex chromatin. This unique system of differential gene regulation are not well understood, and currently little is known about the molecular components and structure of the Barr body itself [9]. This process of inactivation of $\mathrm{X}$ chromosome is known as "Lyonization" [1] [2] [9].

\section{Shape of Appendages}

The inactive $\mathrm{X}$ chromosome in neutrophils appears in one of the five forms (Figures 1-5). They are drumsticks, racquet forms, sessile nodules, small clubs and minor lobes [1] [4] [6] [7]. Big care must be taken to distinguish not sex specific appendages from true drumsticks. These non-specific appendages are distinguished from drumsticks by their size and shape, and/or their less intense staining (drumsticks, like Barr bodies, are highly condensed deeply staining bodies). The majority of such non-specific appendages (small clubs) are smaller than drumsticks (less than $1 \mu$ in diameter); they may be intensely stained, and in such cases can be distinguished from true drumsticks only by size. More rarely, an appendage of the size of a drumstick may have an unstained centre ("tennis racket”) or an apparently larger appendage shows uneven staining, and generally

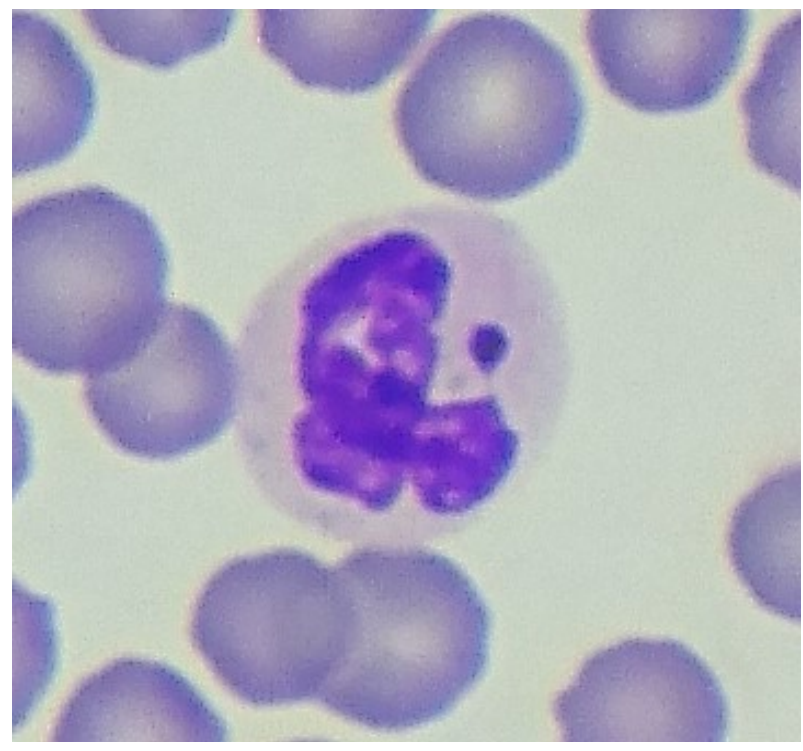

Figure 1. A drumstick appendage in cats neutrophil. 


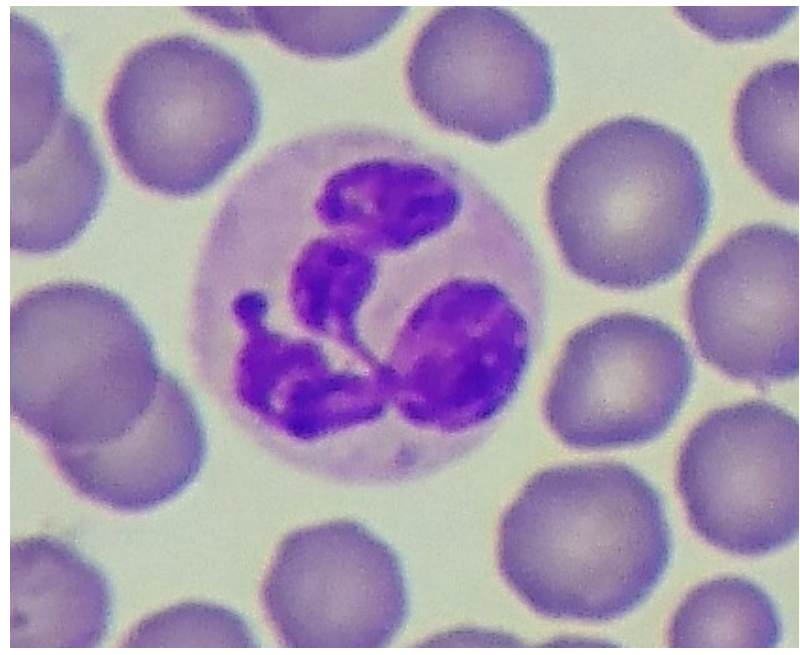

Figure 2. Sessile nodule appendage in cats neutrophil.

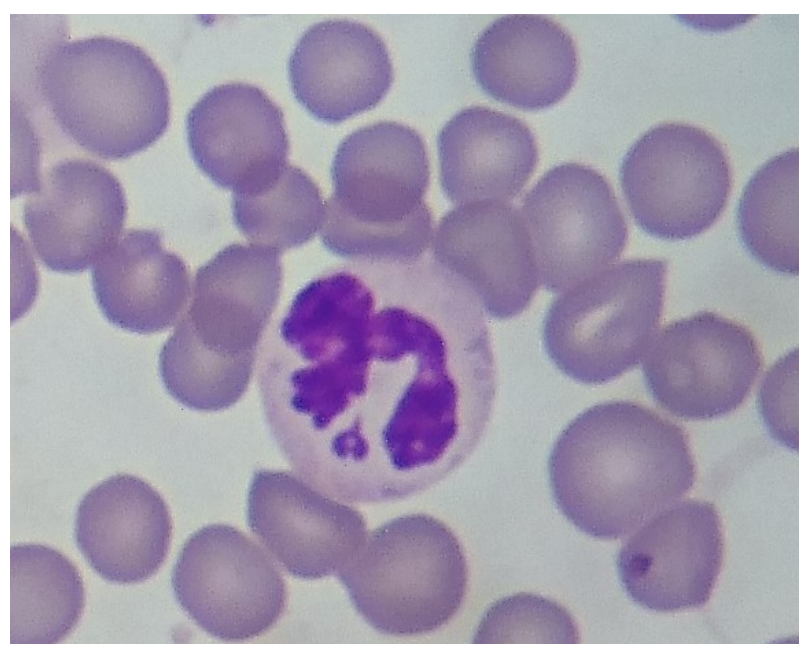

Figure 3. Racquet form appendage in cats neutrophil.

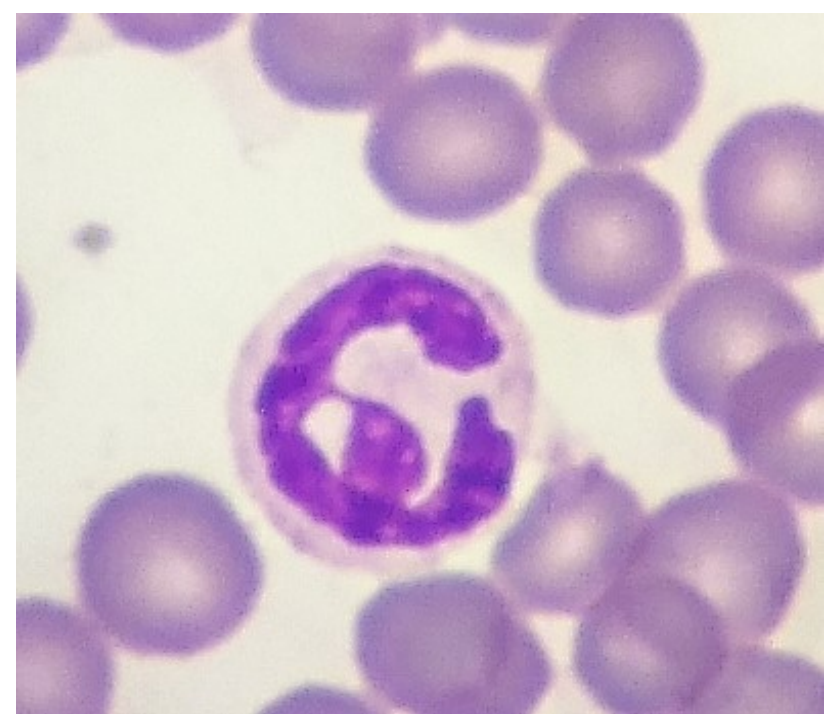

Figure 4. Minor lobe in cats neutrophil. 


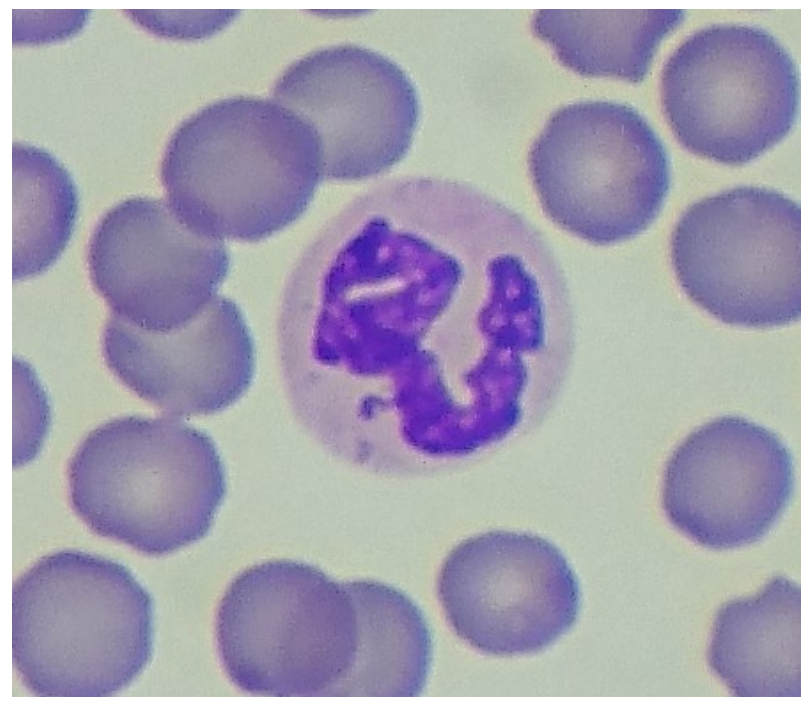

Figure 5. Small club appendage in cats neutrophil.

also a more or less uneven outline (minor lobes). Minor lobes may be connected to the rest of the nucleus by one or two filaments. A proportion of neutrophils of female origin generally contain another type of appendage, consisting of a head which is attached more or less directly to the nucleus. Such a structure is known as a sessile nodule. A sessile nodule may be regarded as an incompletely developed drumstick; and varying degrees of development may be observed [3]. Mehes [10] investigated that drumsticks and sessile nodules are heterochromatic and that other forms are euchromatic.

\section{Drumsticks}

Drumsticks are present in peripheral blood of individuals carrying more than one X-chromosome in at least part of their granulocytes [4]. A drumstick consists of a small nuclear mass of about $1.5 \mu$ in diameter, attached to the body of the nucleus by means of a thin stalk [1] [2] [3] [6] [7] [8] [11]. Some of these drumsticks are hidden or partly hidden by one of the lobes of the nucleus, but must be counted only those which are discrete [7]. The characteristic drumsticks are a feature of the lobulated nucleus of the mature neutrophil, are rarely found in the unsegmented forms, and have not been demonstrated in the precursory cells [7] [11]. More highly lobed cells having a higher incidence of drumsticks [3] [12]. The average incidence of drumsticks is about 1 per 38 neutrophils in the blood films from females, and they are absent in males [3]. The incidence of drumsticks vary between $1 \%$ to $17 \%$ with an average of $2.9 \%$ [1] [3]. These variations could be under different circumstances: during the menstrual cycle, after the administration of adrenocorticotropic hormone and insulin, with senility, and with cachexia [8].

\section{Investigations}

Brahimi et al. (2013) [8] in their investigation used Kosenow's formula where 
nuclear appendages were classified into neutrophils nuclei with drumsticks, sessile nodules, or other appendages like tag and hook. In this study, neutrophils bear the same amount of appendages in both sexes and neutrophilic nuclear appendages were seen in nearly same frequency both in female and male subjects [4] [8]. This fact was confirmed by Verma and Adinarayan investigation (2017) [4]. Results showed that numbers of drumsticks were greater in females than in males [4] [8]. These results confirm the fact that drumstick count is certainly related to sex. They found that drumstick appendages can be seen in males and investigated drumsticks in $0.5 \%$ to $2 \%$ of their polymorphs. In males they found smaller drumsticks and named these projections "drumstick-like" because their structures are similar to typical drumsticks in shape but generally smaller in size [8]. Confirmation of the inactive $\mathrm{X}$ chromosome in the drumstick and the $\mathrm{Y}$ chromosome in the drumstick-like chromosome has been provided by fluorescence in situ hybridization [5] [8]. Females with an isochromosome of the long arms of the $\mathrm{X}$ chromosome have larger and more frequent drumsticks, whereas females with deletions from the $\mathrm{X}$ chromosome have smaller drumsticks [8].

Tupakula et al. (2014) [1] found that the mean number of drumsticks including non-specific appendages and true drumsticks is significantly higher in females $(8.4 \%)$ than in males $(5 \%)(\mathrm{p}<0.001)$. The mean number of true drumsticks were found to be significantly higher in females (7.6\%) than in males (3.9\%) $(\mathrm{p}<0.001)$. The various types of non-specific appendages observed were sessile nodules, minor lobes racket structures and small clubs. The percentage incidence of minor lobes $(46.2 \%)$, racket structures $(42.3 \%)(\mathrm{p}<0.001)$ and small clubs (11.5\%) were more in males when compared to females and sessile nodules $(62.3 \%)(\mathrm{p}<0.001)$ were observed only in females. Combinations of drumstick and sessile nodule presented higher incidence in females whereas combinations of drumstick and rocket structures/small clubs/minor lobes were high in males. It was observed that most of true drumstick head size was not less than $1.1 \mu$ among both the sexes. When the head size of true drumsticks and non-specific appendages were analysed separately it pointed out that less than 1.0 micron diameter head size is in non-specific appendages group only.

In Chatterjee study (2014) [6], 100\% of female subjects and $0.2 \%$ of males exhibited drumstick bodies $(\mathrm{p}<0.0001)$. Few male subjects in this study demonstrated cellular chimerism due to the presence of neutrophilic drumsticks. This finding was reported earlier by Davidson implying that the cells ancestors can be grafted in initial stages of embryogenesis due to the influence of female sex hormones and so affect cellular lineages [13]. He found drumstick as a predominant feature in identification of cytological sex [6].

The majority of investigators believe that "sessile nodules" are as equally sexspecific as drumsticks, but they are more difficult to recognize since they might mingle with the nucleus during a manual spreading of the blood film [8]. Although sex specific, sessile nodules are more easily confused with nonspecific appendages than are drumsticks, and the error inherent in their determination is 
greater [3]. Because of this, only drumstick is considered for sex diagnosis [8].

\section{Drumsticks in Different Mammals}

During sex chromatin investigations sex difference is found in the interphase nuclei of many mammals. Drumsticks are present in certain animals but not in others. In the various groups of animals, there appears to be a close correlation between the presence or absence of drumsticks with that of Barr bodies. Thus drumsticks have been demonstrated in primates, carnivores, and hoofed animals, but none have been found in rodents in which both sexes have nonspecific appendages. Drumsticks are present in the rabbit but difficult to detect. The reason why sex chromatin is clearly visible in some animals and not in others, or only in certain cells of some animals, is not yet known [3].

\section{Hormone Therapy}

It had been reported that an increase in the incidence of small clubs and hooks could be observed after intensive androgen treatment in humans and animals, for example of female patients receiving chronic androgen therapy for mammary carcinoma or of a woman with an androgen-producing tumor of the adrenal gland. These observations prove that the increase in the number of nuclear small clubs and hooks appendages might be a manifestation of a high androgen level in men, too [8] [10]. Although the appearance of these figures may be associated with certain diseases, it should not be regarded as a specific sign for a sigle pathologic or physiologic condition [10].

\section{Genetical Point}

From a genetical point of view, the most important application of sex chromatin testing has been in establishing the incidence of abnormalities of the sex chromosomes [3]. Also in individuals suffering from abnormalities of sex chromosome composition, the correlation between phenotypic sex and sex chromatin body frequency in nuclei may not hold up. These cases are comparatively rare in the population, but could lead to misinterpretation in medicolegal work. Discrepancies between phenotypic sex and chromatin body frequency may be observed in chromatin negative females, chromatin positive males and genetic mosaics [5].

The polymorphonuclear leukocytes of normal male and female infants show the same morphologic sex characteristics as leukocytes from adult males and females respectively. This observation militates against the possibility that the sex chromatin appendage may be a difference in the manifestation of diseases among males and females and strengthens the thesis of genetic origin [11].

\section{Differences in Tumorous Diseases}

Tomonaga et al. (1961) [12] found that the drumstick counts of the neutrophils in chronic granulocytic leukemia are abnormally low when the disease is in 
relapse, increase rapidly during treatments, and return to normal in remission. Good correlation was observed between the number of drumsticks counted and the number of lobes of the segmented leukocytes both in normal subjects and patients with chronic granulocytic leukemia. This relationship exists in spite of the reduced number of drumsticks found in the cells of patients with chronic granulocytic leukemia.

Smetana et al. (2017) [14] investigated that the heterochromatin condensation state of the Barr body in female chronic myeloid leukemia patients was similar to that with silent genes in other nuclear regions. Similarly, the Barr body was also stable and not influenced by the anti-leukemic therapy.

\section{Conclusions}

Accordance researching studies neutrophils nuclei have five forms appendages but only drumstick appendage is sex-specific and considered for sex diagnosis. For sex determination, it was found that drumsticks are significantly higher in females than in males.

Recording of sex chromatin has gained importance in human genetics for establishing the relationship with X-chromosome and determination of the sex of individual has great medico-legal importance to solve many criminal and civil problems [4]. Post mortem blood is good for up to about 6 hours after death for this determination because the leucocytes undergo lysis and degradative changes quite soon after death [5].

Karyotyping, fluorescent in situ hybridization, and PCR are considered confirmatory methods for sex determination. However, use of routine cytological smears with various staining techniques is more feasible and cost-effective [2].

\section{Authors' Contributions}

RI performed the literature review and drafted the manuscript. ZM contributed to the final manuscript. All authors read and approved the final manuscript.

\section{Authors' Information}

$\mathrm{ZM}$ is lecturer and a veterinary hematology-toxicology specialist in animal diseases. Her $\mathrm{Ph}$. D. thesis and postdoctoral research focuses on blood hematological and serum biochemical parameters variability depending on age, gender and the physical condition in žemaitukai horse.

$\mathrm{RI}$ is student on veterinary hematology-toxicology residency, and she is focused on researches of sex chromatin (drumsticks) in cats peripheral blood neutrophils.

\section{Conflict of Interests}

The authors declare that they have no conflict of interests. 


\section{References}

[1] Tupakula, S., Devi Velichety, S. and Thyagaraju, K. (2014) Sexual Dimorphism in Morphology and Morphometry of Neutrophil Drumsticks. International Journal of Anatomy and Research, 2, 720-726. https://doi.org/10.16965/ijar.2014.510

[2] Dahiya, K. (2016) To Evaluate and Compare Barr Bodies and Davidson Bodies-A Forensic Study. Journal of Innovative Dentistry, 6, 1-2.

[3] Mittwoch, U. (1964) Sex Chromatin. Journal of Medical Genetics, 1, 50-76. https://doi.org/10.1136/jmg.1.1.50

[4] Verma, R. and Adinarayan, M. (2017) Reliability of Neutrophilic Nuclear Appendages in Morphological Sex Differentiation. International Journal of Medical Science and Public Health, 6, 783-785.

[5] US Government Printing Office. (1984) Sourcebook in Forensic Serology, Immunology, and Biochemistry. Unit VIII. Determination of Sex of Origin, Nongenetic Markers and Blood Component Profiling. 663-668.

[6] Chatterjee, S. (2014) Reliability of Sexual Dimorphism in Blood. Indian Journal of Physiology and Pharmacology, 58, 400-402.

[7] Davidson, W.M. and Smith, D.R. (1954) A Morphological Sex Difference in the Polymorphonuclear Neutrophil Leucocytes. The BMJ, 2, 2-4, 6-7. https://doi.org/10.1136/bmj.2.4878.6

[8] Brahimi, M., Adda, A., Lazreg, H., Beliali, H., Osmani, S. and Bekadja, M.A. (2013) Can Sex Be Determined from a Blood Smear? Turkish Journal of Hematology, 30, 53-57. https://doi.org/10.4274/tjh.2011.0015

[9] Hong, B., Reeves, P., Panning, B., Swanson, M.S. and Yang, T.P. (2001) Identification of an Autoimmune Serum Containing Antibodies against the Barr Body. Proceedings of the National Academy of Sciences of the United States of America, 98, 8703-8708. https://doi.org/10.1073/pnas.151259598

[10] Méhes, K. (1966) Brief Note Nuclear Projections in Neutrophils. Blood, 28, 598-601.

[11] Tenczar, F.J. and Streitmatter, D.E. (1956) Sex Difference in Neutrophils. American Journal of Clinical Pathology, 26, 384-387. https://doi.org/10.1093/ajcp/26.4.384

[12] Tomonaga, M., Matsuura, G., Watanabe, B., Kamochi, Y. and Ozono, N. (1961) Leukocyte Drumsticks in Chronic Granulocytic Leukemia and Related Disorders. Blood, 18, 581-591.

[13] Booth, P.B., Plaut, G., James, J.D., Ikin, E.W., Moores, P., Sanger R. and Race, R.R. (1957) Blood Chimerism in a Pair of Twins. The BMJ, 1, 1456-1458. https://doi.org/10.1136/bmj.1.5033.1456

[14] Smetana, K., Mikulenková, D. and Klamová, H. (2017) Morphometric and Densitometric Analysis of Heterochromatin during Cell Differentiation Using the Leukaemic Granulocytic Lineage as a Convenient Model. Folia Biologica (Praha), 63, 1-5. 